\title{
Wnt5a and Wnt11 as acute respiratory distress syndrome biomarkers for severe acute respiratory syndrome coronavirus 2 patients
}

To the Editor:

Coronavirus disease 2019 (COVID-19), caused by severe acute respiratory syndrome coronavirus 2 (SARS-CoV-2) infection has spread globally, resulting in declaration of pandemic emergency [1]. COVID-19 patients suffer from various symptoms of infection, including pneumonia, acute respiratory distress syndrome (ARDS) and sepsis. Some known antiviral drugs, including remdesivir, have been proposed as effective agents for the treatment of SARS-CoV-2 infection [2, 3]. Along with the development of potential therapeutics, there is urgency to mitigate the transmission and economic crisis of SARS-CoV-2 via identification of biomarkers that can rapidly indicate the severity of the disease in infected patients. Wnt ligands are secreted glycoproteins and their downstream signalling plays a pivotal role in embryonic development and tissue homeostasis. With remarkable progress in the immunology field, Wnt signalling has gained much attention as a critical regulator in various inflammatory diseases. A large body of evidence has suggested that Wnt ligands are secreted by immune cells, such as peripheral blood mononuclear cells (PBMCs) and non-immune cells, including stroma cells, to regulate inflammatory response and immune cell modulation [4-7]. In addition to their roles in inflammation, studies have reported that these Wnt ligands play key roles in tissue damage and repair [6]. Interestingly, prior studies have reported significant alterations in Wnt5a and Wnt11 expression compared to other Wnt ligands by analysing sera of patients with severe sepsis or sepsis mouse models [4, 8]. Wnt5a signalling has been known to activate in sepsis or ARDS and play a pivotal role in lung inflammation and fibrosis [5, 9], whereas Wnt11 protein has been reported to suppress induction of inflammatory cytokines by regulating NF- $\kappa \mathrm{B}$ activity $[10,11]$. Previous reports have demonstrated that Wnt5a and Wnt11 have opposite functions to one another in response to inflammation $[12,13]$; hence it is thought that Wnt5a has pro-inflammatory effect and Wnt11 may be anti-inflammatory. Therefore, we focused on Wnt5a and Wnt11 to explore their potential relevance to COVID-19-related diseases. In this study, we report Wnt5a and Wnt11 as reliable biomarkers for monitoring of pathological progression in SARS-CoV-2 patients.

Whole blood was collected from admitted SARS-CoV-2 patients at Yeungnam University Medical Center (Daegu, Republic of Korea) when these patients were diagnosed with the SARS-CoV-2 infection at the public health centre in Daegu. None of the patients had taken any medications nor used any mechanical devices upon admission to the hospital. The study protocol (YUH 2020-03-057, 2020-05-031-001) was approved by the institutional review board of Yeungnam University Hospital.

The concentrations of Wnt5a, Wnt11 or cytokines in SARS-CoV-2 patient plasma was quantified according to the manufacturer's instructions using a commercially available ELISA kit. Human recombinant WNT11 protein (H00007481-P01; Abnova, Aachen, Germany), anti-Wnt5a antibody (MAB645; R\&D Systems, Minneapolis, MN, USA), anti-Wnt11 antibody (ab31962; Abcam, Cambridge, MA, USA), Human Protein Wnt-5a ELISA Kit (MBS2886311; MyBioSource, San Diego, CA, USA), and Human Protein Wnt-11 ELISA Kit (MBS281148; MyBioSource) were used.

Heparinised blood samples were used fresh within $4 \mathrm{~h}$, and PBMCs were separated from blood using Ficoll-paque $^{\text {TM }}$ PLUS (17-1440-02, GE Healthcare, Uppsala, Sweden) or NycoPrep (PROGEN

@ERSpublications

Wnt5a/Wnt11 can be used as potential ARDS biomarkers for SARS-CoV-2 patients https://bit.ly/ 3lxEGRA

Cite this article as: Choi $\mathrm{EY}$, Park $\mathrm{HH}$, Kim $\mathrm{H}$, et al. Wnt5a and Wnt11 as acute respiratory distress syndrome biomarkers for severe acute respiratory syndrome coronavirus 2 patients. Eur Respir J 2020; 56: 2001531 [https://doi.org/10.1183/13993003.01531-2020]. 
Biotechnik, Oslo, Norway). Following this, more refined PBMCs were obtained via MACSprep ${ }^{\mathrm{TM}}$ Human PBMC Isolation Kit (130-115-169, Miltenyi Biotec, Bergisch Gladbach, Germany). To verify the effect of Wnt5a-neutralising antibodies or recombinant human Wnt11 on the suppression of cytokine secretion and NF- $\mathrm{BB}$ activation, PBMCs isolated from SARS-CoV-2 patients were incubated with the Wnt5a antibody $\left(20 \mu \mathrm{g} \cdot \mathrm{mL}^{-1}\right)$ or recombinant human Wnt11 $\left(10 \mathrm{ng} \cdot \mathrm{mL}^{-1}\right)$ for $6 \mathrm{~h}$. The supernatant was used for analysis of cytokines ELISA, and the lysate was used for NF- $\kappa \mathrm{B}$ activity analysis using an ELISA-based NF- $\kappa$ B family transcription factor assay kit (43296; Active Motif, Carlsbad, CA, USA). All experiments were performed independently at least three times. Statistically significant differences were determined using unpaired t-tests. Prism software (GraphPad, La Jolla, CA, USA) was used for statistical analyses.

In order to establish reliable diagnostic biomarkers, we conducted a prior study exploring clinical manifestations and various risk factors on severe SARS-CoV-2 patients admitted to Yeungnam University Medical Center [14, 15]. We conducted research to discover new biomarkers in blood based on patient information such as age, body mass index and comorbidities (figure 1a). The SARS-CoV-2 patient blood plasma samples were divided according to the severity of the disease: normal individuals (control group, tested for SARS-CoV-2 infection, but negative); SARS-CoV-2 patients; SARS-CoV-2 patients with ARDS (SARS-CoV-2 ARDS); and individuals discharged after hospitalisation for SARS-CoV-2 infection. ELISA analysis showed a marginal difference in the Wnt5a secretion level between the SARS-CoV2 infection and the control group. Irrespectively, the Wnt5a protein level was dramatically increased in the blood of SARS-CoV-2 ARDS (figure $1 \mathrm{~b}(\mathrm{i})$ ). Interestingly, the Wnt5a protein level was rescued in discharged individuals (figure $1 \mathrm{~b}(\mathrm{i})$ ). This was consistent in the patients who survived, where the Wnt5a level remained low in the plasma, but significant a high level of Wnt5a was still observed in the dead patients, thus demonstrating correlation of the Wnt5 level with the severity of the disease (figure $1 \mathrm{~b}(\mathrm{ii})$ ). In contrast, Wnt11 protein level was robustly induced in the plasma of SARS-CoV2 patients and discharged individuals, but remained at normal levels in SARS-CoV-2 ARDS, where the Wnt5a level was detected at its highest (figure $1 \mathrm{~b}(\mathrm{iii})$ and (iv)).

To assess the regulation of Wnt5a and Wnt11 expression by SARS-CoV-2, PBMCs were isolated from SARS-CoV-2, SARS-CoV-2 ARDS and discharged patients. Based on transcriptional analysis using real-time quantitative PCR, it was observed that the secretion in each plasma sample is associated with differential WNT5a and WNT11 mRNA expression level in the PBMCs (figure 1b(v) and (vi)). Likewise, Wnt5 level was significantly higher and Wnt11 level was significantly lower in the SARS-CoV-2 ARDS patients. Similar restoration in the Wnt5 level and a high level of Wnt11 were observed in discharged patients (figure $1 \mathrm{~b}(\mathrm{v})$ and (vi)). These results suggest that an increased level of Wnt5a is associated with the severity of the disease in SARS-CoV-2 ARDS patients, while a low level of Wnt11 is related with insufficient capability to suppress and alleviate the inflammatory cytokine-induced SARS-CoV-2. To further confirm our findings and expand to clinical significance, PBMCs isolated from normal, SARS-CoV-2, SARS-CoV-2 ARDS and discharged patients were cultured for immunocytochemical analysis. The patient PBMCs were immunostained with specific antibodies and a high level of Wnt5a expression was observed in the SARS-CoV-2 ARDS patients, while less was detectable for Wnt11 (figure 1c(i)). Indeed, PBMCs isolated from SARS-CoV-2 ARDS showed a greater increase in Wnt5a protein secretion than other groups (figure 1c(ii)). Conversely, Wnt11 protein secretion remained at minimum level in PBMCs isolated from SARS-CoV-2 ARDS, and a dramatic increase in Wnt11 protein secretion was observed in PBMCs isolated from discharged individuals (figure 1c(iii)). The effects of Wnt5a and Wnt11 on anti-inflammatory responses were further investigated. Patient PBMCs were treated with anti-Wnt5a neutralising antibody or recombinant ( $\mathrm{r}$ )Wnt11. NF- $\kappa \mathrm{B}$ activation analysis demonstrated that treatment with anti-Wnt5a antibody does not significantly reduce the anti-inflammatory response in the PBMCs of SARS-CoV-2 ARDS (figure 1d(i)). Moreover, secretion of various cytokines, including interleukin (IL)-6, IL-1 $\beta$, IL-4, interferon- $\gamma$ and tumour necrosis factor- $\alpha$ was not significantly altered upon anti-Wnt5a antibody treatment (figure 1d(ii-vii)). However, treatment of SARS-CoV-2 ARDS PBMCs with rWnt11 showed a dramatic inhibitory effect on NF- $\kappa B$ activation as well as on cytokine production (figure $1 \mathrm{~d}(\mathrm{ii}-\mathrm{vii})$ ). These results suggest that Wnt11 protein has great efficacy in reducing inflammatory responses caused by SARS-CoV-2 infection, but Wnt5a is unlikely to be the potential therapeutic target. Previously, Wnt5a expression was upregulated by transforming growth factor (TGF)- $\beta$, which induces pulmonary fibrosis [9], and a recent study has reported a significant increase in TGF- $\beta$ in sera of COVID-19 patients [16]. Thus, it is possible that elevation of Wnt5a in sera of patients with severe COVID-19 may be due to progression of TGF-mediated lung injury, whereby Wnt5a inhibition may not be effective in recovery from inflammatory responses.

In summary, we conducted a single-centre observational study in South Korea to identify biomarkers that could be used to monitor the progression and severity of disease in SARS-CoV-2 patients. By analysing 


\begin{tabular}{|c|c|c|c|c|}
\hline a) & All patients & SARS-CoV-2 ARDS & SARS-CoV-2 & $\begin{array}{c}\text { Discharged } \\
\text { (from SARS-CoV-2) }\end{array}$ \\
\hline $\begin{array}{l}\text { Patients } \\
\text { Characteristics }\end{array}$ & 105 & 25 & 80 & $\begin{array}{c}25 \\
37.9 \pm 14.7\end{array}$ \\
\hline Age years & $45.6 \pm 16.2$ & $67.3 \pm 15.4$ & $52.4 \pm 11.9$ & $23.7 \pm 2.6$ \\
\hline $\mathrm{BMI}$ & $23.5 \pm 3.9$ & $22.8 \pm 4.0$ & $23.4 \pm 3.8$ & \\
\hline \multicolumn{5}{|l|}{ Comorbidities } \\
\hline Cardiovascular disease & $11(10.5)$ & $0(0)$ & $11(13.8)$ & $0(0)$ \\
\hline Cerebrovascular disease & $2(1.9)$ & $0(0)$ & $2(1.9)$ & $0(0)$ \\
\hline Chronic lung disease\# & $3(2.9)$ & $0(0)$ & $3(3.8)$ & $0(0)$ \\
\hline Dementia & $3(2.9)$ & $0(0)$ & $3(3.8)$ & $0(0)$ \\
\hline Diabetes mellitus & $9(8.6)$ & $3(12.0)$ & $6(7.5)$ & $5(20.0)$ \\
\hline Hypertension & $30(28.6)$ & $5(20.0)$ & $25(31.3)$ & $10(40.0)$ \\
\hline Liver disease & $1(0.9)$ & $0(0)$ & $1(1.3)$ & $0(0)$ \\
\hline Malignancy & $4(3.8)$ & $1(4.0)$ & $3(4.2)$ & $0(0)$ \\
\hline Parkinson's disease & $1(0.9)$ & $1(4.0)$ & $0(0)$ & $0(0)$ \\
\hline \multicolumn{5}{|l|}{ Clinical outcomes } \\
\hline Remained in hospital & $62(59.1)$ & $7(28.0)$ & $55(68.8)$ & \\
\hline Discharged & $25(23.8)$ & $0(0)$ & $25(31.2)$ & \\
\hline Died & $18(17.1)$ & $18(72.0)$ & $0(0)$ & \\
\hline
\end{tabular}

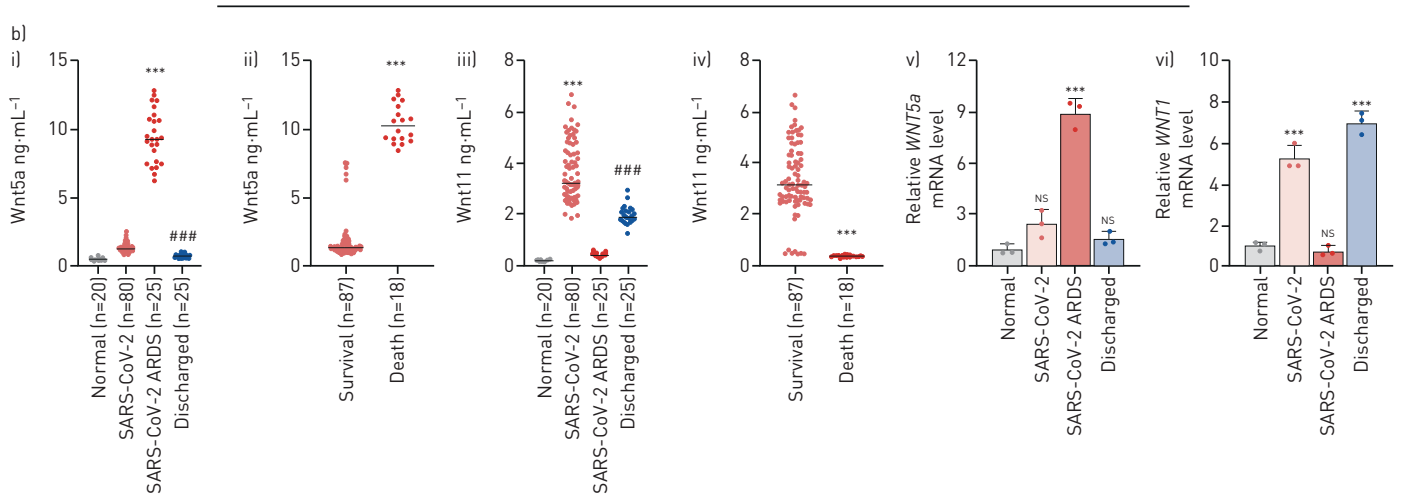

c) $\quad$ Normal PBMCs
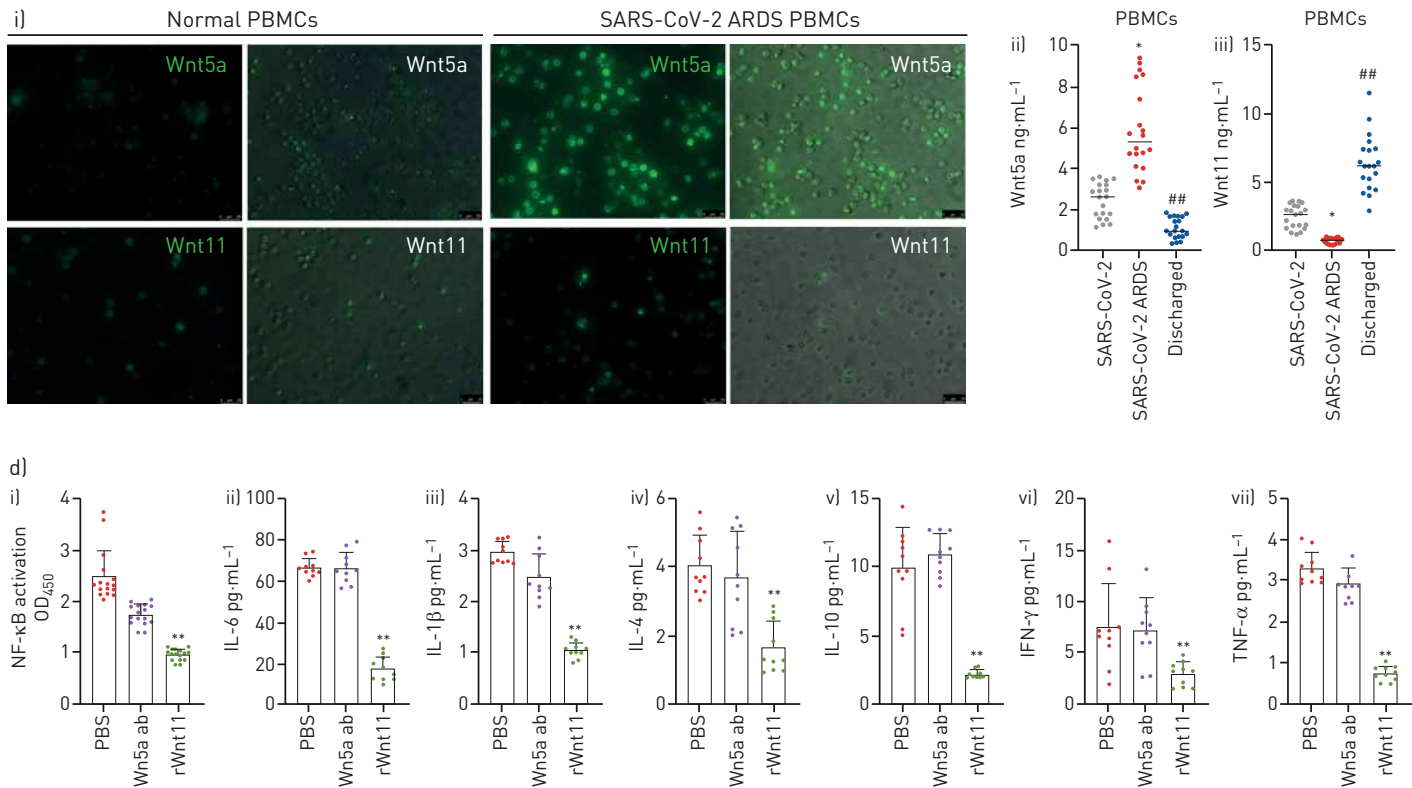

FIGURE 1 Wnt5a/Wnt11: a promising diagnostic marker for severe acute respiratory syndrome coronavirus 2 (SARS-CoV-2) acute respiratory distress syndrome (ARDS). Plasma was secured from 20 healthy volunteers, 80 SARS-CoV-2 patients, 25 patients who progressed to ARDS (SARS-CoV-2 ARDS) and 25 discharged patients (no SARS-CoV-2 detected). a) Baseline characteristics and clinical outcomes of COVID-19 patients admitted to Yeungnam University Hospital (Daegu, Republic of Korea). Data are presented as $\mathrm{n}$, mean \pm SD or $\mathrm{n}$ (\%). BMI: body mass index. ${ }^{\#}$ : includes COPD, asthma, bronchiectasis and interstitial lung disease. b) i-iv) Analysis of Wnt5a and Wnt11 concentrations in SARS-CoV-2 patients; b) $\mathrm{V}$, vil the mRNA expression of Wnt5a and Wnt11 in peripheral blood mononuclear cells (PBMCs) was quantified using quantitative reverse transcriptase-PCR. ${ }^{* * *}: p<0.001$ versus normal or survival; ${ }^{\# \# \#: ~} p<0.001$ versus SARS-CoV-2. c) i) Wnt5a or Wnt11 protein was visualised in PBMCs isolated from normal and SARS-CoV-2 ARDS patients by immunofluorescence staining ( $\times 200)$; c) ii, iii) Wnt5a or Wnt11 secreted from SARS-CoV-2, SARS-CoV-2 ARDS and discharged patients were detected by ELISA. SARS-CoV-2 sepsis patient plasma was incubated with Wnt5a antibody $\left(20 \mu \mathrm{g} \cdot \mathrm{mL}^{-1}\right)$ or recombinant human Wnt11 $\left(10 \mathrm{ng} \cdot \mathrm{mL}^{-1}\right)$ for $6 \mathrm{~h}$ (each group $\left.\mathrm{n}=18\right)$. *: $\mathrm{p}<0.05$ versus SARS-CoV-2; \#\# : $\mathrm{p}<0.01$ versus SARS-CoV-2 ARDS. d) i) Binding activity of NF- $\mathrm{KB}$ (p65) in PBMCs and d) ii-vii) plasma cytokine levels in SARS-CoV-2 ARDS patient PBMCs treated with anti-Wnt5a antibody or recombinant human Wnt11. Data are presented as mean \pm SEM. IL: interleukin; IFN: interferon; TNF: tumour necrosis factor. Significance was set at $p<0.05 .{ }^{* *}$ : $p<0.01$ versus PBS. 
plasma and PBMCs from patients with different pathological severities, our findings reveal that Wnt5a and Wnt11 show opposite expression patterns in SARS-CoV-2 ARDS patients. Based on our results, the measurement of Wnt5a levels in SARS-CoV-2 ARDS patients may be a good indicator for poor prognosis, whereas Wnt11 levels may be a good indicator for ability to survive the disease. Given that Wnt11, not Wnt5a, efficiently inhibits inflammatory responses and cytokine production, it could be exploited as a therapeutic target for the treatment of SARS-CoV-2 ARDS patients.

Eun Young Choi ${ }^{1,9}$, Hee Ho Park ${ }^{2,9}$, Hyelim Kim ${ }^{3,9}$, Hong Nam Kim ${ }^{4,5}$, Inyoung Kim ${ }^{6}$, Soyoung Jeon ${ }^{6}$, Wantae Kim ${ }^{6,10}$, Jong-Sup Bae ${ }^{7,10}$ and Wonhwa Lee $\oplus^{8,10}$

${ }^{1}$ Division of Pulmonary and Allergy, Dept of Internal Medicine, College of Medicine, Yeungnam University and Respiratory Center, Yeungnam University Medical Center, Daegu, Republic of Korea. ${ }^{2}$ Dept of Biotechnology and Bioengineering, Kangwon National University, Chuncheon, Republic of Korea. ${ }^{3}$ College of Pharmacy, Chungnam National University, Daejeon, Republic of Korea. ${ }^{4}$ Center for BioMicrosystems, Brain Science Institute, Korea Institute of Science and Technology (KIST), Seoul, Republic of Korea. ${ }^{5}$ Division of Bio-Medical Science and Technology, KIST School, Korea University of Science and Technology, Seoul, Republic of Korea. ${ }^{6}$ Dept of Biochemistry, College of Natural Sciences, Chungnam National University, Daejeon, Korea. ${ }^{7}$ College of Pharmacy, CMRI, Research Institute of Pharmaceutical Sciences, BK21 Plus KNU Multi-Omics based Creative Drug Research Team, Kyungpook National University, Daegu, Republic of Korea. ${ }^{8}$ Aging Research Center, Korea Research Institute of Bioscience and Biotechnology, Daejeon, Republic of Korea. ${ }^{9}$ These authors contributed equally to this work. ${ }^{10}$ These authors contributed equally to this article as lead authors and supervised the work.

Correspondence: Wonhwa Lee, Aging Research Center, Korea Research Institute of Bioscience and Biotechnology, 125 Gwahak-ro, Yuseong-gu, Daejeon, 34141, Korea. E-mail: bywonhwalee@gmail.com

Received: 2 May 2020 | Accepted after revision: 21 Aug 2020

Acknowledgements: We thank the patients who participated in this study.

Author contributions: E.Y. Choi, H.H. Park, W. Kim, J-S. Bae and W. Lee designed and directed the study. H. Kim, H. N. Kim and W. Lee carried out ELISA, Western blot, immunocytochemistry and cytokine assays. E.Y. Choi collected blood samples from patients. E.Y. Choi, J-S. Bae, I. Kim, S. Jeon and W. Lee directed the data analysis. H.H. Park, W. Kim and W. Lee wrote the manuscript. All authors reviewed the manuscript and consented to the description of author contributions.

Conflict of interest: None declared.

Support statement: This work was supported by a grant from the National Research Foundation of Korea (NRF) funded by the Ministry of Education (NRF-2018R1D1A1B07050422 and NRF-2018R1C1B600274913). This work was supported by the National Research Foundation of Korea (NRF) grant funded by the Korea government (MSIT) (NRF-2020R1A2C1004131). This research was supported by a grant of the Korea Health Technology R\&D Project through the Korea Health Industry Development Institute (KHIDI), funded by the Ministry of Health \& Welfare, Republic of Korea (grant number: HI15C0001). Funding information for this article has been deposited with the Crossref Funder Registry.

\section{References}

1 Phua J, Weng L, Ling L, et al. Intensive care management of coronavirus disease 2019 (COVID-19): challenges and recommendations. Lancet Respir Med 2020; 8: 506-517.

2 Grein J, Ohmagari N, Shin D, et al. Compassionate use of remdesivir for patients with severe Covid-19. N Engl J Med 2020; 382: 2327-2336.

3 Wang M, Cao R, Zhang L, et al. Remdesivir and chloroquine effectively inhibit the recently emerged novel coronavirus (2019-nCoV) in vitro. Cell Res 2020; 30: 269-271.

4 Gatica-Andrades M, Vagenas D, Kling J, et al. WNT ligands contribute to the immune response during septic shock and amplify endotoxemia-driven inflammation in mice. Blood Adv 2017; 1: 1274-1286.

5 Villar J, Cabrera-Benítez NE, Ramos-Nuez A, et al. Early activation of pro-fibrotic WNT5A in sepsis-induced acute lung injury. Crit Care 2014; 18: 568.

6 Staal FJ, Luis TC, Tiemessen MM. WNT signalling in the immune system: WNT is spreading its wings. Nat Rev Immunol 2008; 8: 581-593.

7 Chae WJ, Bothwell ALM. Canonical and non-canonical Wnt signaling in immune cells. Trends Immunol 2018; 39 : 830-847.

8 Pereira C, Schaer DJ, Bachli EB, et al. Wnt5A/CaMKII signaling contributes to the inflammatory response of macrophages and is a target for the antiinflammatory action of activated protein $\mathrm{C}$ and interleukin-10. Arterioscler Thromb Vasc Biol 2008; 28: 504-510.

9 Newman DR, Sills WS, Hanrahan K, et al. Expression of WNT5A in idiopathic pulmonary fibrosis and its control by TGF- $\beta$ and WNT7B in human lung fibroblasts. J Histochem Cytochem 2016; 64: 99-111.

10 Liu X, Wu S, Xia Y, et al. Wingless homolog Wnt11 suppresses bacterial invasion and inflammation in intestinal epithelial cells. Am J Physiol Gastrointest Liver Physiol 2011; 301: G992-G1003.

11 Morishita Y, Kobayashi K, Klyachko E, et al. Wnt11 gene therapy with adeno-associated virus 9 improves recovery from myocardial infarction by modulating the inflammatory response. Sci Rep 2016; 6: 21705.

12 Sato A, Kayama H, Shojima K, et al. The Wnt5a-Ror2 axis promotes the signaling circuit between interleukin-12 and interferon- $\gamma$ in colitis. Sci Rep 2015; 5: 10536.

13 Railo A, Nagy II, Kilpeläinen P, et al. Wnt-11 signaling leads to down-regulation of the Wnt/ $\beta$-catenin, JNK/AP-1 and NF-KB pathways and promotes viability in the CHO-K1 cells. Exp Cell Res 2008; 314: 2389-2399. 
14 Hong KS, Lee KH, Chung JH, et al. Clinical features and outcomes of 98 patients hospitalized with SARS-CoV-2 infection in Daegu, South Korea: a brief descriptive study. Yonsei Med J 2020; 61: 431-437.

15 Jang JG, Hur J, Choi EY, et al. Prognostic factors for severe coronavirus disease 2019 in Daegu, Korea. J Korean Med Sci 2020; 35: e209.

16 Shen B, Yi X, Sun Y, et al. Proteomic and metabolomic characterization of COVID-19 patient sera. Cell 2020; 182 : $59-72$.

Copyright (C)ERS 2020 .

This version is distributed under the terms of the Creative Commons Attribution Non-Commercial Licence 4.0. 